\title{
EVOLUTIONARY HELIUM AND CNO ANOMALIES IN THE ATMOSPHERES AND WINDS OF MASSIVE HOT STARS
}

\author{
Nolan R. Walborn \\ Space Telescope Science Institute* \\ 3700 San Martin Drive \\ Baltimore, Maryland 21218
}

\begin{abstract}
The ubiquitous evidence for processed material in the atmospheres, winds, and circumstellar ejecta of massive stars will be reviewed. A broad array of normal and peculiar evolutionary stages is considered, up to and including Type II supernova progenitors. The quantitative analysis of these spectra is difficult, and until recently for the most part only qualitative or approximate results have been available. However, several important current programs promise reliable abundance determinations, which will enable detailed comparisons with recent evolutionary calculations. A significant emerging result is that the morphologically normal majority of both hot and cool supergiants may already display an admixture of CNO-cycle products in their atmospheres. It may become possible in this way to identify blue supergiants returning from the red supergiant region, as appears to have been the case for the SN 1987A progenitor.
\end{abstract}

\section{INTRODUCTION}

In this review I wish to develop two themes. First, that the evidence for processed material at the surfaces of evolving massive stars is virtually everywhere; in fact it is now reasonable to hypothesize that all massive stars display at least the products of hydrogen burning prior to their finales as Type II (or Ib?) supernovae. Second, that current progress both in the quantitative observation of abundance anomalies, and in their computation from theoretical evolutionary models, promises an imminent and significant synthesis. Consistent with the nature of the observational evidence, this review will be concerned essentially with effects of the CNO cycle, that is the enhancement of helium and nitrogen at the expense of hydrogen, carbon, and oxygen, with one exception (the WC stars). A wide range of specific topics is addressed, encompassing the OBN/OBC and related objects, including mass-transfer binaries; morphologically normal OB stars; the Luminous Blue Variables, including Eta Carinae; briefly, the Wolf-Rayet stars and late-type supergiants; a survey of the pertinent evolutionary calculations; and finally, Type II supernova progenitors, including that of SN 1987A. Evolutionary chemical anomalies will be seen to exist in the stellar atmospheres, winds, and/or ejected circumstellar material of all these classes of objects.

\section{OBN/OBC STARS AND RELATED OBJECTS}

\section{A. Background}

Two nitrogen-deficient, early-B supergiants described exactly twenty years ago by the Jascheks (1967) were the first known cases of CNO anomalies in OB absorption-line spectra. The first examples of the inverse phenomenon, OB spectra with enhanced nitrogen, were reported by Walborn (1970), and the OBN/OBC classification dichotomy was introduced shortly thereafter (Walborn 1971). While this notation has mnemonic value, it does incur the subtle disadvantage of suggesting, by analogy with the WR classification, a very advanced evolutionary state for the OBC stars. Such an implication was not intended, since the OBC stars, all supergiants, have hydrogen

* Operated by AURA, Inc. under contract with NASA. 
lines of normal strength and in fact, as further discussed in the next section, the most likely interpretation is that they are less evolved than their morphologically normal relatives. A general review of the OBN/OBC categories and their possible interpretations was presented by Walborn (1976). Walborn and Panek (1985) showed that the optical anomalies are reflected in the ultraviolet stellar-wind features as well, significantly extending the ionization range over which they are seen in a given spectrum, and supporting their interpretation in terms of abundance effects (see also Walborn, Nichols-Bohlin, and Panek 1985).

\section{B. Current Programs}

Two important current programs are contributing to alleviate the scarcity of quantitative abundance determinations for the OBN/OBC stars. Schönberner et al. (1987) have analyzed the optical spectra of four OBN and related dwarf or giant objects, finding the very significant result that not only the $\mathrm{N} / \mathrm{C}$ ratios but also the $\mathrm{He} / \mathrm{H}$ are larger than normal, thus establishing beyond a reasonable doubt that $\mathrm{CNO}$-cycled material is indeed being observed. Wollaert, Lamers, and de Jager (1987) have compared equivalent widths in the ultraviolet spectra of fourteen OBN/OBC stars of all luminosity classes to those of normal stars, providing limits on the abundance anomalies by means of a curve-of-growth analysis.

\section{Individual Objects}

\section{1. $H D 99840$}

This star is a new, extreme BN supergiant, the discovery of which I find quite pleasing. As described above, the OBN/OBC spectral anomalies were first detected optically, and correlated anomalies were later found to exist in the ultraviolet. These latter effects have now in turn permitted the discovery of the BN nature of HD 93840, through the careful analysis of Savage and Massa (1987). The UV spectrum shows a very strong N V P Cyg profile, with little or no corresponding C IV emission, which is highly abnormal (Walborn and Nichols-Bohlin 1987). Savage and Massa classified the star as BN1 II-Ib on the basis of the UV spectrum and previous optical types, while N. Houk classified it B1/2 Iab/b in the Michigan HD Catalogue. Its galactic latitude is $+11^{\circ}$, in keeping with the relatively high latitudes of the OBN class (Walborn 1970; Bolton and Rogers 1978; Bisiacchi, López, and Firmani 1982). Further investigation of the optical spectrum is planned for the coming season.

\section{2. $H D 157098$}

This interesting supergiant was described optically as related to the OBN category, because while silicon and carbon appear normal, the strength of nitrogen would correspond to a higher temperature and that of magnesium to a lower (Walborn 1976, 1980). A quantitative optical analysis has been performed by Lennon and Dufton (1986), revealing enhanced nitrogen and helium abundances, which led them to suggest that this object may be evolving blueward following a red supergiant phase. Recently, J. Nichols-Bohlin and I in collaboration with D. Lennon and colleagues at Belfast have observed the 1200-1900 $\AA$ spectrum of HD 157038 at high resolution in a very long exposure with the International Ultraviolet Explorer. The stellar-wind features correspond well to a normal B3 Ia spectrum, which is perhaps not surprising since they are mostly due to $\mathrm{C}$ and $\mathrm{Si}$ at that type. A detailed analysis will be undertaken by Lennon et al.

\section{Mass-Transfer Binaries}

It is possible that all OBN objects are a result of either active or previous mass transfer in close binary systems (Bolton and Rogers 1978). Two exceptionally interesting active systems, both of $\beta$ Lyrae type, are HD 72754 and HD 163181. The optical spectrum of the former, including the nitrogen anomaly, was extensively discussed by Thackeray (1971), and a UV analysis is promised by de Freitas Pacheco and Codina Landaberry (1986). The anomalous optical CNO spectrum of HD 163181 was discovered by Walborn (1972) and analyzed by Kane, McKeith, and Dufton (1981). A brief description of the UV spectrum has been given by Hutchings and van Heteren (1981). In collaboration with J. Nichols-Bohlin, I have inspected the IUE high-resolution data and find an anomalously large ratio of N V/C IV stellar-wind features, together with some indications of spectral variability. 
Significantly, Balachandran, Lambert, Tomkin, and Parthasarathy (1986) have recently established that the spectrum of $\beta$ Lyrae itself reveals the presence of CNO-cycle products. Its spectral type is too late for ready morphological detection of CNO anomalies in the blue-violet, but the quantitative analysis has shown that it is physically related to the $\mathrm{BN}$ binaries. Furthermore, $\mathrm{Pe}-$ ters and Polidan (1984) have found evidence for extreme carbon deficiency in the high-temperature accreting material of a number of Algol-type binary systems. Hence it may be that not only are all OBN objects mass-transfer binaries, but many other mass-transfer binaries are related to the OBN category.

\section{MORPHOLOGICALLY NORMAL OB STARS}

\section{A. Supergiants}

All of the O9-B0 supergiants in the Orion Belt $(\delta, \epsilon$, and $\varsigma$ Orionis) and in NGC 6231 (Scorpius OB1) have systematically nitrogen-deficient spectra, relative to the majority of supergiants of the same types (Walborn 1976). A prior result of nitrogen deficiency among the NGC 6231 mainsequence members, which would have favored an explanation in terms of initial abundances at formation, has recently been withdrawn (Brown et al. 1986b). An interpretation of the supergiant anomaly in terms of evolutionary processes is therefore indicated. The hypothesis proposed by Walborn (1976) was that the morphologically normal majority of OB supergiants actually display some fraction of CNO-cycled material at their surfaces, while the rarer, nitrogen-weak OBC objects, such as those in the two associations, are relatively less evolved and have physically normal (i.e. main-sequence) surface abundances. This hypothesis receives substantial support from the very important current results of Bohannan, Voels, Abbott, and Hummer (1987), who find normal helium abundances in $\delta$ and $\zeta$ Orionis, but enhanced helium in the morphologically normal 09.5 Ia standard $\alpha$ Camelopardalis. These determinations corroborate the pioneering study by Baschek, Kodaira, and Scholz (1972), who found that the atmosphere of the morphologically normal 09.5 Iab supergiant HD 188209 contains an admixture of $20-40 \%$ of CNO-cycled material, relative to that of $\zeta$ Orionis. Kudritzki et al. (1987) have derived significant overabundances of helium and nitrogen in the LMC supergiants Sanduleak $-65^{\circ} 21$ (09.5 Ia) and $-68^{\circ} 41$ (B0.5 Ia), and suggested that they are post-red supergiants; the spectra of these two objects are definitely morphologically normal, as one can easily see by comparing the high-quality digital data of Fitzpatrick (1987) to the spectrograms of OBN supergiants with similar types shown by Walborn $(1971,1972)$. Similarly, both Kudritzki, Simon, and Hamann (1983) and Bohannan, Abbott, Voels, and Hummer (1986) have found an enhanced helium abundance in $\zeta$ Puppis using state-of-the-art model-atmosphere techniques. Bohannan et al. (1987) have also suggested that the systematically smaller gravity effect shown by $P$. Conti's helium equivalent-width measures in $O$-type spectra, as compared to that for hydrogen, could be due to compensation by higher helium abundances in the supergiants. While quantitative analysis for a larger sample is certainly desirable before definitive conclusions are drawn, nevertheless the hypothesis that all OB supergiants mix CNO-cycle products into their atmospheres must now be considered very seriously.

\section{B. Main-Sequence Stars}

CNO anomalies in main-sequence stars naturally suggest origins in initial conditions or mass transfer. However, Lyubimkov $(1977,1984)$ has presented surprising, detailed evidence for trends of increasing helium and nitrogen abundances with age in main-sequence and giant $O B$ stars. Such an effect would present a new challenge for interpretation. On the other hand, the recent results of Brown et al. (1986a, b) do not appear to show these trends. Currently, D. R. Gies and D. L. Lambert are obtaining new, high-quality observational material for a welcome, independent investigation of this important question.

\section{LUMINOUS BLUE VARIABLES}

This term is now used to encompass the Hubble-Sandage, $\mathrm{S}$ Doradus, and $\mathrm{P}$ Cygni variables, 
as well as $\eta$ Carinae as an extreme case, for all of which there is an emerging unity of phenomenology and interpretation. These objects lie along the Humphreys-Davidson (1979) limit in the HR diagram, which evidently corresponds to an instability leading to episodic shell ejection and curtailing further redward evolution of the most massive stars. It is probable that this phenomenon represents the transition from $O B$ to WN stages, at least for masses between about 50 and 100 $M_{\odot}$.

\section{A. HS, S Dor, P Cyg Stars}

Evidence for an enhanced nitrogen abundance and/or a deficiency of carbon and oxygen in P Cygni itself was presented by Luud (1967), and in the related southern object AG Carinae by Caputo and Viotti (1970). Lamers, de Groot, and Cassatella (1983) discussed P Cygni as an object evolving between red supergiant and WN stages. The $\lambda 4600$ regions in the beautiful and remarkably similar spectra of the HS stars AF Andromedae in M31 and Variable B in M33 shown by Kenyon and Gallagher (1985) are dominated by P Cyg profiles of the N II multiplet at 4601.5, $4607.2,4613.9,4621.4,4630.5$, and $4643.1 \AA$, contrary to statements by the authors. These spectra are essentially identical to those of P Cygni and AG Carinae (in 1976) themselves. The spectrum of the LMC S Dor-type variable HDE 269006 at minimum shown by Wolf, Appenzeller, and Stahl (1981) has the same multiplet dominating in absorption. More detailed analyses of all these spectra are likely to prove rewarding and demonstrate significant overabundances of nitrogen. Enhanced nitrogen abundances have also been found in the probably related objects HD 38489 in the LMC by Shore and Sanduleak (1983), and S18 in the SMC by Shore, Sanduleak, and Allen (1987).

I have been particularly interested in a category of LMC objects now designated Ofpe/WN9, which combine high-excitation Of characteristics with lower-excitation emission and $P$ Cyg spectral features (Walborn 1977). One of these objects, HDE 269858 (=R127) has subsequently undergone an outburst followed in detail by Stahl et al. (1983), Stahl and Wolf (1986a), and Wolf, Stahl, Smolinski, and Cassatella (1987), and it is now regarded as the hottest known S Dor variable. An important result of high-resolution observations of these objects with the Cerro Tololo 4-meter echelle spectrograph was the discovery of velocity-doubled forbidden lines, implying nonspherical circumstellar nebulae, for which an overabundance of nitrogen and an underabundance of oxygen were found (Walborn 1982). Stahl (1987) has resolved two of these shells by direct imagery, while Stahl and Wolf (1986b) have found spectroscopic evidence for similar structures around a wider range of related LMC objects, including HDE 269006 and S Doradus itself.

Recently, Leitherer and Zickgraf (1987) have detected the circumstellar shell of P Cygni by direct imaging, finding evidence for an abnormally large [N II]/H $\alpha$ ratio. Two other objects which are probably galactic counterparts of the LMC Ofpe/WN9 stars are HD 148937 (Walborn 1973) and AG Carinae (Stahl 1986); both have spectacular ejected circumstellar nebulae. Leitherer and Chavarría (1987) have established a nitrogen overabundance in the HD 148937 nebula (NGC 61648165). The AG Carinae nebula has been described by Thackeray (1977) and Viotti, Cassatella, Ponz, and The (1987); it may well be nitrogen-rich, but an abundance analysis remains to be done.

\section{B. Eta Carinae}

This famous object may be considered the most spectacular LBV, on the basis of its luminosity and outburst magnitude. Its location in a giant $\mathrm{H}$ II region is also atypical, and its mass is probably substantially greater than $100 M_{\odot}$. From the fact that no such object is observed in 30 Doradus, despite the much larger early $O$ and $W R$ populations, one may infer that it represents a very short-lived phase. Because of the spectroscopic continuity among the O3 If and WN-A stars in the Carina Nebula (Walborn 1974), I suspect that Eta Carinae is a post-WNL object. An important step toward understanding its nature was the discovery that the expanding knots in its complex outer shell are nitrogen- and helium-rich (Davidson, Walborn, and Gull 1982; Davidson, Dufour, Walborn, and Gull 1986). These knots have simpler optical and ultraviolet nebular spectra than the denser inner regions, displaying five ionization stages of $\mathrm{N}$ and none of $\mathrm{C}$ or $\mathrm{O}$. The helium overabundance has been nicely corroborated by the infrared line analysis of Allen, Jones, and Hyland (1985). The compositions and velocities of the knots are highly reminiscent of the quasistationary flocculi in Cassiopeia $\mathrm{A}$, believed to be circumstellar material ejected by the progenitor star, making Eta Carinae a strong presupernova candidate. 


\section{WOLF-RAYET STARS}

I shall cite only briefly the most significant recent results bearing on the chemical compositions of these key objects, since they are the subject of other papers at this meeting which promise further advances. The WR stars are probably the first to have revealed evolutionary abundance anomalies, but unfortunately the same extravagance which facilitated their discovery has obstructed quantitative analysis. A new idea which has the markings of a breakthrough toward a definitive model is that of very high underlying surface temperatures, which may be capable of ionizing and driving the envelopes radiatively (Cherepashchuk, Eaton, and Khaliullin 1984; Pauldrach, Puls, Hummer, and Kudritzki 1985; D. Abbott, private communication).

The status of WR abundance analyses through IAU Symposium 99 was very well summarized by Willis (1982); see also Linda J. Smith and Willis $(1982,1983)$. Since then, additional information on the $\mathrm{He} / \mathrm{H}$ ratios has been provided by Conti, Leep, and Perry (1983), while substantial progress on modeling the He spectra has been contributed by Hamann and Schmutz (1987 and references therein) and by Hillier (1987a, b). The WC stars are special, as the only well-defined class of massive stars showing us the products of helium burning. Currently, improved determinations of their C/He ratios are being made by Lindsey F. Smith and Hummer (1987) from near infrared spectra, and by Torres (1987) optically.

A number of WN stars also have circumstellar ring nebulae, for which abundance determinations are of obvious relevance. Nitrogen and helium enrichments have been demonstrated in several of them by Talent and Dufour (1979), Contini and Shaviv (1980), and Kwitter (1981, 1984).

\section{LATE-TYPE SUPERGIANTS}

This review is about hot massive stars, but at least up to about $50 M_{\odot}$ they are either destined to become or have already been late-type supergiants. Hence for completeness, and as a preliminary to the final two sections below, a brief survey of the impressive evidence for CNO-cycle products in the atmospheres of the latter is also of interest. An enhancement of nitrogen and/or deficiency of carbon, and in some cases anomalous isotope ratios of $\mathrm{C}$ and $\mathrm{O}$, have been observed in the following objects and classes: Cepheids and related nonvariable supergiants (Luck and Lambert 1981, 1985); Canopus (Desikachary and Hearnshaw 1982); Betelgeuse and Antares (Harris and Lambert 1984; Lambert, Brown, Hinkle, and Johnson 1984); and Polaris (Luck and Bond 1986).

\section{EVOLUTIONARY CALCULATIONS OF SURFACE ABUNDANCES}

An extensive array of theoretical calculations of evolving surface abundances is available for comparison with the observations discussed in the previous sections. Intermediate-mass stars up to $11 M_{\odot}$ have been considered by Becker and Iben $(1979,1980)$ and Becker and Cox $(1982)$; the mechanism is convective dredge-up, and the results are applicable to the Cepheids and related supergiants. For massive stars, stellar-wind mass loss becomes the key mechanism; results for 15-150 $M_{\odot}$ single stars have been computed and in several cases compared with observations by Stothers and Chin (1979); Noels, Conti, Gabriel, and Vreux (1980); Gabriel and Noels (1981); Noels and Gabriel (1981); Brunish and Truran (1982a, b); Doom (1982a, b); Dearborn and Blake (1984); Pylyser, Doom, and de Loore (1985); Prantzos, Doom, Arnould, and de Loore (1986); and Langer and El Eid (1986). Rotationally induced mixing has been considered by Maeder (1987b). Results for mass-transfer binaries are presented by Vanbeveren and Doom (1980); Vanbeveren (1982, 1983); and Doom and de Grève (1983).

The most detailed charting of evolving surface abundances in the HR diagram for massive stars has been carried out by Maeder (1983; 1987a) and Maeder and Meynet (1987). In principle, very fine comparisons with the observations are enabled; the precision of the latter is now the limiting factor, but that situation is currently improving as discussed above. An enormous amount of theoretical information is made usefully accessible by this work, about which you will hear more in the following talk. I wish to emphasize only a few points here. Stars with initial masses between 12 and about $20 M_{\odot}$ will explode as red supergiants, after revealing CNO-cycle products in their cool 
atmospheres. Stars with masses between about 20 and $50 M_{\odot}$ return from the red supergiant region, displaying CNO-cycled material during their blueward evolution, and becoming blue-supergiant or possibly WR supernova progenitors. This history most likely describes SN 1987A. Finally, stars more massive than about $50 M_{\odot}$ reveal processed material in their atmospheres soon after leaving the main sequence, and they never reach a red supergiant stage in agreement with observation (Humphreys and Davidson 1979). Thus all supernova progenitors are expected to have CNO-cycle products in their atmospheres or circumstellar environments, except perhaps for those very massive objects which reach a WC stage. It should be noted in passing that there may also be an upper mass limit for the latter, not yet reflected in these models, since few or no WC stars are observed in giant H II regions (see also Langer 1987).

\section{TYPE II SUPERNOVA PROGENITORS}

The observations are in excellent accord with the above expectation: in every instance for which information is available, there is evidence for CNO-cycled material at the surfaces or in the vicinities of massive supernova progenitors. The classical case is the Cassiopeia A remnant, which consists of nitrogen-rich, relatively slow-moving "quasi-stationary flocculi", and oxygenrich, fast-moving knots; the former have been interpreted as circumstellar material ejected by the progenitor, while the latter are interior debris from the explosion itself (Peimbert and van den Bergh 1971; Chevalier and Kirshner 1978; Lamb 1978). Recently, fast-moving nitrogen-rich knots have been discovered at the periphery of the Cas $A$ remnant, which are consistent with explosive ejection from the stellar surface (Fesen, Becker, and Blair 1987). Puppis A has now been shown to have a dichotomous composition/kinematical structure of nitrogen-rich, slow and oxygen-rich, fast material entirely analogous to that of Cas A (Winkler and Kirshner 1985). Nitrogen-rich filaments have also been found in W50, the remnant associated with SS433 (Kirshner and Chevalier 1980 and references therein). The remnant of Kepler's supernova may provide yet another example (van den Bergh and Kamper 1977; Dennefeld 1982; Bandiera 1987). It would be of considerable interest to search for nitrogen-rich features near other young SNR.

But the evidence is not limited to remnants; nitrogen-rich material has in fact been detected in three Type II SN in action. Fransson et al. (1984) derived a very large N/C ratio from the ultraviolet spectrum of $1979 \mathrm{C}$ in M100 shortly after maximum. Niemela, Ruíz, and Phillips (1985) observed a WN-like optical spectrum from 1983K in NGC 4699 in unprecedented pre-maximum coverage.

And now there is SN 1987A in the Large Magellanic Cloud for our delight and edification. So far as is known, the best spectroscopic observations of the progenitor star, Sanduleak $-69^{\circ} 202$ (Walborn, Lasker, Laidler, and Chu 1987), consist of $460 \AA / \mathrm{mm}$ and $110 \AA / \mathrm{mm}$ (at $\mathrm{H} \gamma$ ) objective-prism plates obtained at the European Southern Observatory, the B3 I classification being based upon the latter (Rousseau et al. 1978). González et al. (1987) have investigated the lower-resolution material in comparison with another, normal B3 Ia object in the LMC observed similarly, finding that a blend of $\mathrm{N}$ II and He I lines is considerably stronger in the progenitor spectrum. Unfortunately, the interpretation is ambiguous, since the effect could be due either to a chemical anomaly or to a slightly earlier spectral type (say B2) of $\mathrm{Sk}-69^{\circ} 202$. The higher-resolution material confirms that the features in question are stronger in the latter (L. Prévot, private communication), but without resolving the composition/temperature ambiguity as yet; further investigation of these important spectrograms is planned. Meanwhile, definitive evidence for nitrogen-rich material associated with SN $1987 \mathrm{~A}$ is provided by the current far-ultraviolet, narrow emission-line spectrum reported by Wamsteker, Gilmozzi, Cassatella, and Panagia (1987) and Kirshner et al. (1987). This spectrum most likely arises in circumstellar material being excited by the outburst, and it is extremely similar to those of the $\eta$ Carinae knots (Davidson, Walborn, and Gull 1982; Davidson, Dufour, Walborn, and Gull 1986). Hence, the direct observation of Type II supernovae confirms the presence of CNO-cycled material in or around the progenitors, as predicted by both the observations and theory of massive stars reviewed here.

Thanks to Dorothy Whitman for her consistently outstanding manuscript support. 


\section{REFERENCES}

Allen, D. A., Jones, T. J., and Hyland, A. R. 1985, Ap. J. 291, 280.

Balachandran, S., Lambert, D. L., Tomkin, J., and Parthasarathy, M. 1986, M.N.R.A.S. 219, 479.

Bandiera, R. 1987, Ap. J. $319,885$.

Baschek, B., Kodaira, K., and Scholz, M. 1972, Ap. Letters 12, 227.

Becker, S. A. and Cox, A. N. 1982, Ap. J. 260, 707.

Becker, S. A. and Iben, I. Jr. 1979, Ap. J. 232, 831. . 1980, Ap. J. 237, 111.

Bisiacchi, G. F., López, J. A., and Firmani, C. 1982, Astr. Ap. 107, 252.

Bohannan, B., Abbott, D. C., Voels, S. A., and Hummer, D. G. 1986, Ap. J. 308, 728.

Bohannan, B., Voels, S. A., Abbott, D. C., and Hummer, D. G. 1987, I.A.U. Symposium 132.

Bolton, C. T. and Rogers, G. L. 1978, Ap. J. 222, 234.

Brown, P. J. F., Dufton, P. L., Lennon, D. J., Keenan, F. P., and Kilkenny, D. 1986a, Astr. Ap. $155,113$.

Brown, P. J. F., Dufton, P. L., Lennon, D. J., and Keenan, F. P. 1986b, M.N.R.A.S. 220, 1003.

Brunish, W. M. and Truran, J. W. 1982a, Ap. J. 256, 247. .1982b, Ap. J. Suppl. 49, 447.

Caputo, F. and Viotti, R. 1970, Astr. Ap. 7, 266.

Cherepashchuk, A. M., Eaton, J. A., and Khaliullin, Kh. F. 1984, Ap. J. $281,774$.

Chevalier, R. A. and Kirshner, R. P. 1978, Ap. J. 219, 931.

Conti, P. S., Leep, E. M., and Perry, D. N. 1983, Ap. J. 268, 228.

Contini, M. and Shaviv, G. 1980, Astr. Ap. 88, 117.

Davidson, K., Dufour, R. J., Walborn, N. R., and Gull, T. R. 1986, Ap. J. 305, 867.

Davidson, K., Walborn, N. R., and Gull, T. R. 1982, Ap. J. (Letters) 254, L47.

Dearborn, D. S. P. and Blake, J. B. 1984, Ap. J. 277, 783.

de Freitas Pacheco and Codina Landaberry 1986, Rev. Mexicana Astron. Astrof. 12, 184.

Dennefeld, M. 1982, Astr. Ap. 112, 215.

Desikachary, K. and Hearnshaw, J. B. 1982, M.N.R.A.S. $201,707$.

Doom, C. 1982a, Astr. Ap. 116, 303. . 1982b, Astr. Ap. 116, 308.

Doom, C. and de Grève, J. P. 1983, Astr. Ap. 120, 97.

Fesen, R. A., Becker, R. H., and Blair, W. P. 1987, Ap. J. 313, 378.

Fitzpatrick, E. L. 1987, I.A.U. Symposium 132.

Fransson, C., Benvenuti, P., Gordon, C., Hempe, K., Palumbo, G. G. C., Panagia, N., Reimers, D., and Wamsteker, W. 1984, Astr. Ap. 132, 1.

Gabriel, M. and Noels, A. 1981, Astr. Ap. 94, L1.

González, R., Wamsteker, W., Gilmozzi, R., Walborn, N., and Lauberts, A. 1987, ESO SN1987A Workshop.

Hamann, W.-R. and Schmutz, W. 1987, Astr. Ap. 174, 173.

Harris, M. J. and Lambert, D. L. 1984, Ap. J. 281, 739.

Hillier, D. J. 1987a, Ap. J. Suppl. 63, 947. . 1987b, Ap. J. Suppl. 63, 965.

Humphreys, R. M. and Davidson, K. 1979, Ap. J. 232, 409.

Hutchings, J. B. and van Heteren, J. 1981, Pub. A.S.P. 93, 626.

Jaschek, M. and Jaschek, C. 1967, Ap. J. 150, 355.

Kane, L. G., McKeith, C. D., and Dufton, P. L. 1981, M.N.R.A.S. 194, 537.

Kenyon, S. J. and Gallagher, J. S. III 1985, Ap. J. 290, 542.

Kirshner, R. P. and Chevalier, R. A. 1980, Ap. J. (Letters) 242, L77.

Kirshner, R., Sonneborn, G., Cassatella, A., Gilmozzi, R., Wamsteker, W., and Panagia, N. 1987, I.A.U. Circ., No. 4435.

Kudritzki, R. P., Groth, H. G., Butler, K., Husfeld, D., Becker, S., Eber, F., and Fitzpatrick, E. 1987, ESO SN1987A Workshop. 
Kudritzki, R. P., Simon, K. P., and Hamann, W.-R. 1983, Astr. Ap. 118, 245.

Kwitter, K. B. $1981, A$ p. J. 245, 154. . 1984, Ap. J. 287, 840 .

Lamb, S. A. 1978, Ap. J. 220, 186.

Lambert, D. L., Brown, J. A., Hinkle, K. H., and Johnson, H. R. 1984, Ap. J. 284, 223.

Lamers, H. J. G. L. M., de Groot, M., and Cassatella, A. 1983, Astr. Ap. 123, L8.

Langer, N. 1987, Astr. Ap. 171, L1.

Langer, N. and El Eid, M. F. 1986, Astr. Ap. 167, 265.

Leitherer, C. and Chavarría K., C. 1987, Astr. Ap. 175, 208.

Leitherer, C. and Zickgraf, F.-J. 1987, Astr. Ap. 174, 103.

Lennon, D. J. and Dufton, P. L. 1986, Astr. Ap. 155, 79.

Luck, R. E. and Bond, H. E. 1986, Pub. A.S.P. 98, 442.

Luck, R. E. and Lambert, D. L. 1981, Ap. J. 245, 1018. . 1985, Ap. J. 298, 782.

Luud, L. S. 1967, Soviet Astron. 11, 211

Lyubimkov, L. S. 1977, Astrophysics 13, 71. . 1984, Astrophysics 20, 255.

Maeder, A. 1983, Astr. Ap. 120, 113. . 1987a, Astr. Ap. 173, 247. 1987b, Astr. Ap. 178, 159.

Maeder, A. and Meynet, G. 1987, Astr. Ap. 182, 243.

Niemela, V. S., Ruíz, M. T., and Phillips, M. M. 1985, Ap. J. 289, 52.

Noels, A., Conti, P. S., Gabriel, M., and Vreux, J.-M. 1980, Astr. Ap. 92, 242.

Noels, A. and Gabriel, M. 1981, Astr. Ap. 101, 215.

Pauldrach, A., Puls, J., Hummer, D. G., and Kudritzki, R. P. 1985, Astr. Ap. 148, L1.

Peimbert, M. and van den Bergh, S. 1971, Ap. J. 167, 223.

Peters, G. J. and Polidan, R. S. 1984, Ap. J. 283, 745.

Prantzos, N., Doom, C., Arnould, M., and de Loore, C. 1986, Ap. J. 304, 695.

Pylyser, E., Doom, C., and de Loore, C. 1985, Astr. Ap. 148, 379.

Rousseau, J., Martin, N., Prévot, L., Rebeirot, E., Robin, A., and Brunet, J. P. 1978, Astr. Ap. Suppl. 31, 243.

Savage, B. D. and Massa, D. 1987, Ap. J, 314, 380.

Schönberner, D., Herrero, A., Becker, S., Eber, F., Butler, K., Kudritzki, R. P., and Simon, K. P. 1987, Astr. Ap., in press.

Shore, S. N. and Sanduleak, N. 1983, Ap. J. 273, 177.

Shore, S. N., Sanduleak, N., and Allen, D. A. 1987, Astr. Ap. 176, 59.

Smith, L. F. and Hummer, D. G. 1987, preprint.

Smith, L. J. and Willis, A. J. 1982, M.N.R.A.S. 201, 451. . 1983, Astr. Ap. Suppl. 54, 229.

Stahl, O. 1986, Astr. Ap. 164, 321 and 170, 197. . 1987, Astr. Ap. 182, 229.

Stahl, O. and Wolf, B. 1986a, Astr. Ap. 154, 243. 1986b, Astr. Ap. 158, 371.

Stahl, O., Wolf, B., Klare, G., Cassatella, A., Krautter, J., Persi, P., and Ferrari-Toniolo, M. 1983, Astr. Ap. 127, 49.

Stothers, R. and Chin, C.-W. 1979, Ap. J. 233, 267.

Talent, D. L. and Dufour, R. J. 1979, Ap. J. 233, 888.

Thackeray, A. D. 1971, M.N.R.A.S. 154, 103. . 1977, M.N.R.A.S. 180, 95.

Torres, A. V. 1987 , preprint.

Vanbeveren, D. 1982, Astr. Ap. 105, 260. 1983, Astr. Ap. 119, 239. 
Vanbeveren, D. and Doom, C. 1980, Astr. Ap. 87, 77.

van den Bergh, S. and Kamper, K. W. 1977, Ap. J. 218, 617.

Viotti, R., Cassatella, A., Ponz, D., and Thé, P. S. 1987, Astr. Ap., in press.

Walborn, N. R. 1970, Ap. J. (Letters) 161, L149.

. 1971, Ap. J. (Letters) 164, L67.

1972, Ap. J. (Letters) 176, L119.

1973, A. J. 78, 1067.

1974, Ap. J. 189, 269.

1976, Ap. J. 205, 419.

1977, Ap. J. 215, 53.

1980, Ap. J. Suppl. 44, 535.

1982, Ap. J. 256, 452.

Walborn, N. R., Lasker, B. M., Laidler, V. G., and Chu, Y.-H. 1987, Ap. J. (Letters) 321, L41.

Walborn, N. R. and Nichols-Bohlin, J. 1987, Pub. A.S.P. 99, 40.

Walborn, N. R., Nichols-Bohlin, J., and Panek, R. J. 1985, International Ultraviolet Explorer Atlas of O-Type Spectra from 1200 to $1900 \AA$, NASA RP-1155.

Walborn, N. R. and Panek, R. J. 1985, Ap. J. 291, 806.

Wamsteker, W., Gilmozzi, R., Cassatella, A., and Panagia, N. 1987, I.A.U. Circ., No. 4410.

Willis, A. J. 1982, I.A.U. Symposium 99, p. 87.

Winkler, P. F. and Kirshner, R. P. 1985, Ap. J. 299, 981.

Wolf, B., Appenzeller, I., and Stahl, O. 1981, Astr. Ap. 103, 94.

Wolf, B., Stahl, O., Smolinski, J., and Cassatella, A. 1987, Astr. Ap., in press.

Wollaert, J. P. M., Lamers, H. J. G. L. M., and de Jager, C. 1987, Astr. Ap., in press. 